\title{
Performance and Practicality of a Rapid Molecular Test for the Diagnosis of Strep A Pharyngitis in a Remote Australian Setting
}

\author{
Janessa L. Pickering, ${ }^{1,2 \star}$ Dylan D. Barth, ${ }^{1,2}$ and Asha C. Bowen ${ }^{1,2,3,4}$ \\ ${ }^{1}$ Wesfarmer's Centre for Vaccines and Infectious Diseases, Telethon Kids Institute, Nedlands, Australia; ${ }^{2}$ Centre for Child Health Research, \\ University of Western Australia, Nedlands, Australia; ${ }^{3}$ Department of Infectious Diseases, Perth Children's Hospital, Nedlands, Australia; ${ }^{4}$ Menzies \\ School of Health Research, Charles Darwin University, Darwin, Australia
}

\begin{abstract}
Over 5 days, 120 schoolchildren from two schools in the remote Kimberley region of Australia were screened for Strep A pharyngitis. Molecular point-of-care testing identified Strep A pharyngitis in 13/18 (72.2\%) symptomatic children. The portability and feasibility of molecular point-of-care testing was highly practical for remote settings.
\end{abstract}

Delivery of health care in remote settings can be difficult, in part because of a shortage of healthcare professionals and the physical and logistical barriers to service delivery, ${ }^{1}$ resulting in reliance on a mobile healthcare workforce with high turnover. ${ }^{2}$ These barriers underpin inequitable health outcomes for remote-living people compared with urban-dwelling populations. $^{1}$

Traditional culture-based laboratory methods used to diagnose group A streptococcal (Strep A, Streptococcus pyogenes) pharyngitis may take up to 5 days before results become available for clinical care in remote locations in Australia, and may be impossible in many parts of the world where microbiology laboratories are unavailable. ${ }^{3,4}$ Molecular pointof-care tests (PoCTs) offer many advantages for timely clinical management such as reducing the "time to diagnosis" and guiding immediate treatment decisions, ${ }^{5}$ and limiting the underdiagnosis and overdiagnosis of Strep $A$ in populations at risk of serious complications, which may prevent acute rheumatic fever (ARF) and heart disease (RHD) ${ }^{6}$ as well as antibiotic resistance, and antibiotic shortages. ${ }^{7,8}$ We evaluated the performance of the ID NOW PoCT (previously Alere I) ${ }^{9}$ to detect Strep $A$ in an ongoing pharyngitis and impetigo surveillance in remote Australian children. This is the first description of ID NOW testing in remote Australian settings.

This study was nested in a larger, school-based surveillance study for Strep A pharyngitis and impetigo in children aged 5-15 years living in the Kimberley region of Western Australia (WA), Australia (Figure 1). The Kimberley is a large $\left(423,517 \mathrm{~km}^{2}\right)$, sparsely populated region in northern WA with a permanent population of 34,364 (2016 Australian Bureau of Statistics census data). Aboriginal people make up $~ 40.0 \%$ of the Kimberley's population, with the majority living in small, isolated communities of $<1,000$ people throughout the region.

Ethics approval was obtained from the Western Australia Aboriginal Human Research Ethics Committee (WAAHEC, 892), the University of Western Australia (UWA, RA/4/20/5101) Human Research Ethics Committee, Kimberley Aboriginal Health Planning Forum research subcommittee, and the WA Catholic Education Office (RP2018/55). Written informed consent to participate in the surveillance study was obtained from the parent/legal guardian of each participant and verbal assent confirmed from all children before sample collection.

\footnotetext{
*Address correspondence to Janessa L. Pickering, Wesfarmer's Centre for Vaccines and Infectious Diseases, Telethon Kids Institute, 15 Hospital Ave., Nedlands 6009, Australia. E-mail: janessa.pickering@
} telethonkids.org.au
Every consented child present at two primary schools were interviewed and examined for pharyngitis using a checklist of symptoms and clinical decision rules used in low-resource settings. ${ }^{10}$ A photo of the throat was obtained, and the size of the tonsils was graded using the Brodsky grading scale. ${ }^{11}$ Swabs of the tonsillar fossa were collected according to a standard procedure using cotton swabs (Copan, Brescia, Italy); stored in skim milk, soy trypticase, glucose, and glycerol broth (SGGB) (PathWest Media, Perth, Australia); refrigerated; and transported to the Telethon Kids Institute microbiology laboratory (Perth) for storage at $-80^{\circ} \mathrm{C}$ for up to 2 months before culture. . $^{3,12}$

For children with sore throats, a second cotton swab was obtained for PoC testing immediately after the first swab to minimize patient discomfort. The ID NOW machine (Abbott, Chicago, IL) was operated by three study staff as per the manufacturer's instructions. Instructional materials are available online. ${ }^{13}$ Swabs were immediately processed using the ID NOW on-site followed by storage in SGGB and transported to Perth. Staff reports on the practicality of the PoCT were collected using face-to-face interviews.

Swabs were cultured for Strep A using standard laboratory methodology, ${ }^{14}$ on horse blood agar (HBA) and HBA containing colistin and nalidixic acid plates (PathWest Media). Semi-quantitative counts for each sample were recorded as either $\leq 10$ colony-forming units (CFUs), 11-50 CFUs, and $>50$ CFUs but not the predominant colony type, or the predominant colony type. Streptococcus pyogenes was identified with bacitracin sensitivity and latex agglutination testing for all streptococcal groups (Oxoid, Hampshire, United Kingdom).

Statistical analysis was performed using STATA (StataCorp, College Station, TX) to calculate descriptive statistics and test of significance. The Student's $t$-test was used to evaluate the association between the average number of symptoms and the likelihood of a positive Strep A result.

Of 120 children assessed during surveillance, 20 (16.7\%) reported sore throat symptoms (and were later referred to their local healthcare service for treatment). The mean age was 10 years $(S D=2.03)$. There were $5 / 18$ males $(27.8 \%)$, and $12 / 18$ (66.7\%) were Aboriginal. Eighteen of these 20 (90.0\%) provided a swab for PoC testing and 17/18 (94.4\%) for culture. Culture was performed on the stored PoCT swab for this participant only.

Clinical symptoms among the 18 PoCT participants were throat pain $(15,83.3 \%)$, difficulty swallowing $(9,50.0 \%)$, rhinorrhea $(8,44.4 \%)$, cough $(5,27.7 \%)$, hoarseness $(2,11.1 \%)$, 


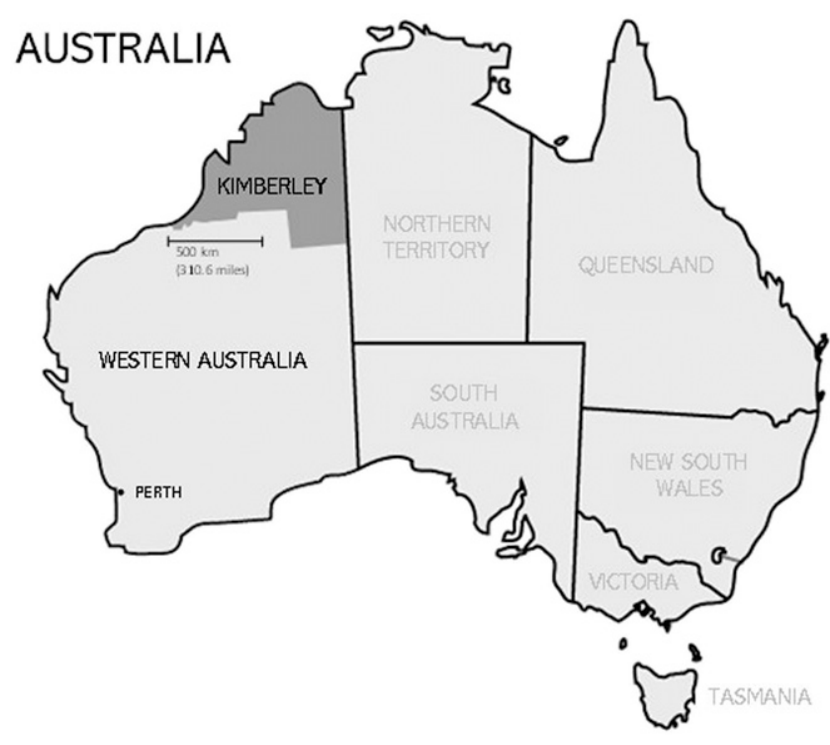

FIgURE 1. A map of Australia showing the Kimberley region in the north of the state of Western Australia. The capital city of the state, Perth, is more than $2,500 \mathrm{~km}$ (1,553 miles) to the south where most culture diagnostics occur.

tonsillar erythema $(9,50.0 \%)$ and swelling $(10,55.5 \%)$, exudate on the pharynx $(3,16.7 \%)$ and tonsils $(3,16.7 \%)$, tender anterior cervical lymph node $(5,27.7 \%)$, and anterior cervical lymph nodes $>1.5 \mathrm{~cm}$ in diameter $(3,16.7 \%)$. Tonsillar hypertrophy grades $1(5,27.7 \%), 2(4,22.2 \%)$, and $3(9,50.0 \%)$ were observed. There was no significant difference in the number of symptoms $(P=0.23)$ between participants with positive PoCTs (mean $=5.1, \mathrm{SD}=2.7$ ) and those who tested negative (mean $=3.3, \mathrm{SD}=2.1$ ). Likewise, there was no significant difference $(P=0.09)$ between participants with positive culture (mean $=5.6, \mathrm{SD}=2.7$ ) and those who had a negative result (mean $=3.5, \mathrm{SD}=2.1$ ).

Strep A was identified in 13/18 (72.2\%) of children by the PoCT and in $9 / 18$ (50.0\%) by culture. Of those positive for Strep A, five samples recorded $<10$ CFUs, three were within 11-50 CFUs, and one was $>50$ CFUs but not the predominant colony type. All five children negative for the PoCT were also negative for Strep A culture. Group C Streptococcus was identified by culture in one child with a positive PoCT, despite no evidence of Strep A on culture, and in another child with a negative PoCT. We found a sensitivity of $100 \%$ and specificity of $55 \%$ for the PoCT with the positive predictive value of $69 \%$ and the negative predictive value of $100 \%$.

Staff reported several benefits of the PoCT technology. Highly technical training was not required, and staff with limited laboratory background were able to perform the test easily. A criticism of the test was the accumulation of biohazard waste from single-use testing plastics and solutions provided with each kit which required external disposal. The PoCT device is small, portable, and easily transported, and a customized carry bag for the device is suggested. Ambient temperatures were up to $35^{\circ} \mathrm{C}$, and maintaining the optimal temperature for the machine $\left(15-30^{\circ} \mathrm{C}\right.$ and $10-80 \%$ relative humidity) was achieved within air-conditioned offices. The processed PoCT swab could be cultured (off-label use) and matched with primary culture results; this may facilitate a single swab in future. Test results were available quickly, aiding face-to-face discussions and treatment referral. The immediate positive test gave staff language to use when speaking with the child about their sore throat in real time. Staff then liaised with the study nurse to notify parents and caregivers within the day to initiate antibiotic treatment in a timely manner.

We report the first results of molecular Strep A PoCTs in remote settings which adds to the urban primary care literature. ${ }^{7,9}$ Before this study, laboratory-based studies have confirmed the value of PoC testing for remote or resourcepoor high-risk populations ${ }^{15}$ where they can be used in the prevention of ARF. In our pilot, we found PoC testing to add value to the diagnosis of Strep A pharyngitis as part of our school-based surveillance activities. PoC testing was more sensitive than culture in identifying Strep A. There was no obvious correlation between symptomatology and PoCTs or culture; however, the number of children with pharyngitis was too low to allow symptom-specific statistical analysis. The value of accurate molecular testing over clinical algorithms for the diagnosis of pharyngitis (less sensitive and specific) has the potential to reduce under and over prescription of antibiotics, with improved patient outcomes (e.g., fewer ARF cases and lower antimicrobial resistance rates). In high-risk settings for ARF and RHD, overdiagnosis is much less of a concern.

Point-of-care testing had the additional benefit of a known Strep A result which facilitated improved patient, parent, and healthcare worker education. Although used in a research setting, the known high sensitivity of this platform facilitated quick and clear communication. In combination with symptoms, the PoCT may help improve early treatment of children with sore throats (and primary prevention of ARF).

Further research in implementation and health economic analyse are needed to progress this toward clinical care. ${ }^{8}$ These efforts must address the need for testing to meet standards, quality control and assurance, and adequate education and training of healthcare professionals. ${ }^{16}$ These barriers can be overcome; staff with limited training could easily use the PoCT machinery, and the test itself has a low false-negative rate. Limiting the cost per test is also essential for PoCT integration.

Our pilot study has some limitations. Children may have reported a sore throat to miss class time, but only one child had a single symptom and was negative on the PoCT. Our sample size was also small. We only used PoCTs for symptomatic children as this was a primary validation study of a test with a low risk of false-positive results.

We report Strep A PoCTs for pharyngitis have value in remote Australian settings where the burden of ARF is high. The use of such tests should be encouraged to guide timely treatment. Further analyses (e.g., health economic evaluation) are needed in Australia to progress this simple technology into clinical care.

Received April 23, 2020. Accepted for publication August 2, 2020.

Published online September 8, 2020.

Acknowledgments: We wish to thank the research study nurse, Robyn Macarthur (Derby Aboriginal Health Service), and surveillance study volunteer, Lee-Ann Fowler (Telethon Kids Institute). We also thank Abbott representatives Thor Cembala and Michelle Williamson for facilitating a loan arrangement for the ID NOW machine. We acknowledge the support and participation of students, staff, and their families at the two schools in the Kimberley, WA.

Financial support: This study was supported by the National Health and Medical Research Council (NHMRC) of Australia through their 
support of the End Rheumatic Heart Disease Centre for Research Excellence (END RHD CRE) (GTN1080401). J. L. P. received support from the Western Australia Department of Health Merit Award and the Western Australia Health Translation Network. A. C. B. receives support from the National Health and Medical Research Council with an Investigator Award (GTN1175509).

Disclosure: No funding was received from Abbott for the conduct, data collection, or analysis of these data. The ID NOW cartridges were purchased with study funds. The ID NOW machine was provided by Abbott for the purpose of the study.

Authors' addresses: Janessa L. Pickering and Dylan D. Barth, Wesfarmer's Centre for Vaccines and Infectious diseases, Telethon Kids Institute, Nedlands, Australia, and Centre for Child Health Research, University of Western Australia, Nedlands, Australia, E-mails: janessa.pickering@ telethonkids.org.au and dylan.barth@telethonkids.org.au. Asha C. Bowen, Wesfarmer's Centre for Vaccines and Infectious diseases, Telethon Kids Institute, Nedlands, Australia, Centre for Child Health Research, University of Western Australia, Nedlands, Australia, Department of Infectious Diseases, Perth Children's Hospital, Nedlands, Australia, and Menzies School of Health Research, Charles Darwin University, Darwin, Australia, E-mail: asha.bowen@health.wa.gov.au.

\section{REFERENCES}

1. Wilson NW, Couper ID, De Vries E, Reid S, Fish T, Marais BJ, 2009. A critical review of interventions to redress the inequitable distribution of healthcare professionals to rural and remote areas. Rural Remote Health 9: 1060.

2. Wakerman J, Humphreys J, Russell D, Guthridge S, Bourke L, Dunbar T, Zhao Y, Ramjan M, Murakami-Gold L, Jones MP, 2019. Remote health workforce turnover and retention: what are the policy and practice priorities? Hum Resour Health 17: 99.

3. Bowen AC, Tong SY, Chatfield MD, Andrews RM, Carapetis JR, 2013. Comparison of three methods for the recovery of skin pathogens from impetigo swabs collected in a remote community of northern territory, Australia. Trans $R$ Soc Trop Med Hyg 107: 384-389.

4. McDonald M, Towers R, Fagan P, McKinnon M, Benger N, Andrews R, Currie BJ, Carapetis J, 2006. Recovering streptococci from the throat, a practical alternative to direct plating in remote tropical communities. J Clin Microbiol 44: 547-552.
5. Hardy V, Thompson M, Alto W, Keppel GA, Hornecker J, Linares A, Robitaille B, Baldwin LM, 2016. Exploring the barriers and facilitators to use of point of care tests in family medicine clinics in the United States. BMC Fam Pract 17: 149.

6. May PJ, Bowen AC, Carapetis JR, 2016. The inequitable burden of group A streptococcal diseases in Indigenous Australians. Med J Aust 205: 201-203.

7. Pritt BS, Patel R, Kirn TJ, Thomson RB Jr., 2016. Pointcounterpoint: a nucleic acid amplification test for Streptococcus pyogenes should replace antigen detection and culture for detection of bacterial pharyngitis. J Clin Microbiol 54: 2413-2419.

8. Bowen AC, Daveson K, Anderson L, Tong SY, 2019. An urgent need for antimicrobial stewardship in Indigenous rural and remote primary health care. Med J Aust 211: 9-11.e1.

9. Berry GJ, Miller CR, Prats MM, Marquez C, Oladipo OO, Loeffelholz MJ, Petersen JR, 2018. Comparison of the Alere i Strep A test and the BD veritor system in the detection of group A Streptococcus and the hypothetical impact of results on antibiotic utilization. J Clin Microbiol 56: e01310-17.

10. Engel ME et al., 2017. The cape town clinical decision rule for streptococcal pharyngitis in children. Pediatr Infect Dis J 36: 250-255.

11. Brodsky L, 1989. Modern assessment of tonsils and adenoids. Pediatr Clin North Am 36: 1551-1569.

12. Hare KM, Stubbs E, Beissbarth J, Morris PS, Leach AJ, 2010. Swab transport in Amies gel followed by frozen storage in skim milk tryptone glucose glycerol broth (STGGB) for studies of respiratory bacterial pathogens. J Microbiol Methods 81: 253-255.

13. Abbott, 2020. ID NOWTM Strep A 2. Available at: https:// www.alere.com/en/home/product-details/id-now-strep-a-2.html. Accessed July 13, 2020.

14. Johnson DK et al., 1996. Laboratory diagnosis of group A streptococcal infections. WHO Collaborating Centre for Reference and Research on Streptococci. Prague, Czech Republic: World Health Organization.

15. Ralph AP, Holt DC, Islam S, Osowicki J, Carroll DE, Tong SYC, Bowen AC, 2019. Potential for molecular testing for group A Streptococcus to improve diagnosis and management in a high-risk population: a prospective study. Open Forum Infect Dis 6: ofz097.

16. Shaw JLV, 2016. Practical challenges related to point of care testing. Pract Lab Med 4: 22-29. 